\title{
Research to meet the needs of the NHS: a review of published NIHR HTA clinical trials
}

\author{
Amanda Young $^{1 *}$, Ruairidh Milne ${ }^{2}$, James Raftery ${ }^{1}$, Louise Dent ${ }^{3}$, David Turner ${ }^{1}$, Peter Davidson ${ }^{1}$, Andrew Cook \\ From Clinical Trials Methodology Conference 2011 \\ Bristol, UK. 4-5 October 2011
}

\section{Background}

The NIHR HTA programme was established in 1993 with the aim of being 'needs-led'. This was achieved through commissioning trials to address questions specified by the programme rather than by applicants. At the time this was an unusual funding model and the programme has developed experience that is now globally significant.

There is dissatisfaction worldwide with the focus of much existing clinical research (the arrival in the USA of Comparative Effectiveness Research is one attempt to address this) and many trials focus on outcomes that are of little relevance to patients [1]. However, apart from early work reviewing the sources of topics suggested to the HTA programme [2], there has been no systematic attempt to review the programme's success in being needs-led.

\section{Objectives}

The establishment of a database of metadata for HTA trials allowed us to examine the experience from a long series of published HTA trials. We therefore set out to review how far trials funded by the NIHR HTA programme were indeed needs-led by looking at:

- The source of the original suggestion

- The priority given by the programme to the research

- The patient-relevance of the primary outcome

\section{Methods}

The study used a variety of methods to assess the extent to which HTA trials published up to March 2011 were needs-led $(\mathrm{n}=112)$. The sources of topic identification and the prioritisation methods were examined over a 10 year period. The type of primary outcome measure

\footnotetext{
* Correspondence: A.J.Young@southampton.ac.uk

'University of Southampton's Wessex Institute, Southampton, SO16 7NS, UK Full list of author information is available at the end of the article
}

reported during the commissioning stage, research protocol and HTA monograph were assessed using Gandhi et al. (2008) [1] classification list.

\section{Results}

The source of the original suggestion: 77/112 trials (69\%) addressed questions that came out of the widespread consultation, a mix of postal and online questionnaires of managers, clinicians and patient and professional groups. Majority of the remaining trials were from systematic reviews $(22 \%, 25 / 112)$.

The priority given by the programme to the research: $58 / 84$ had been recommended as 'must commission'

Table 1 Summary data

\begin{tabular}{cc}
\hline Description & № (\%) \\
\hline Sources used to identify research suggestions: & \\
Widespread consultation & $77(68.8)$ \\
Systematic review / DARE review & $25(22.3)$ \\
Horizon Scanning Centre & $3(2.7)$ \\
Reconsidered / recycled topics & $5(4.5)$ \\
No data available & $2(1.8)$ \\
\hline
\end{tabular}

Priority given by the programme to the research:

(up to and including publication date 1999)

Recommended for commissioning - must commission $\quad 58$ (51.8)

Recommended for commissioning 22 (19.6)

Category unknown (FT and NSCAG) 4 (3.6)

(Post 1999)

Commissioning requested by PSG

Direct commissioning in priority area

$1(0.9)$

Actual type of primary outcome reported in the monograph:

Patient important (including those with additional outcomes) 84 (75.0) Surrogate $7(6.3)$

Physiological / laboratory $1(0.9)$

Other $18(16.1)$

No information available / unable to judge primary outcome 2 (1.8) 
priorities by the HTA programme, meaning that they would be advertised and if that failed to result in funding, further work would be done to ensure that they were taken forward.

The patient-relevance of the primary outcome: Three quarters of trials addressed patient relevant outcomes $(75 \%, 84 / 112)$. This compares with $46 \%$ and $45 \%$ in previous studies [1], [3].

\section{Conclusions}

These analyses suggest that the first 112 published HTA trials can indeed claim to be meeting the information needs of the NHS. Further work is required to compare these results with elsewhere and to develop more robust measures for the future.

\section{Acknowledgements}

The authors would like to thank members of the advisory group for their valuable comments during the development of the metadata database. We also wish to thank the National Institute for Health Research Health Technology Assessment programme for providing the funding for this project.

\section{Author details}

${ }^{1}$ University of Southampton's Wessex Institute, Southampton, SO16 7NS, UK.

${ }^{2} \mathrm{NIHR}$, Evaluation, Trials and Studies Coordinating Centre (NETSCC), University of Southampton, Southampton, SO16 7NS, UK. ${ }^{3}$ University of Southampton Clinical Trials Unit, Southampton General Hospital,

Southampton, SO16 6YD, UK.

Published: 13 December 2011

\section{References}

1. Gandhi G, Murad H, Fujiyoshi A, Mullan R, Flynn D, Elamin M, Swiglo B, Isley W, Guyatt G, Montori V: Patient-important outcomes in registered diabetes trials. JAMA 2008, 299(21):2543-2549.

2. Chase D, Milne R, Stein $K$, Stevens A: What are the relative merits of the sources used to identify potential research priorities for the NHS HTA programme? International Journal of Technology Assessment in Health Care 2000, 16(3):743-750.

3. Rahimi K, Malhotra A, Banning A, Jenkinson C: Outcome selection and role of patient reported outcomes in contemporary cardiovascular trials: systematic review. BMJ 2010, 341:c5707.

doi:10.1186/1745-6215-12-S1-A86

Cite this article as: Young et al:: Research to meet the needs of the NHS: a review of published NIHR HTA clinical trials. Trials 2011 12(Suppl 1):A86.

\section{Submit your next manuscript to BioMed Central} and take full advantage of:

- Convenient online submission

- Thorough peer review

- No space constraints or color figure charges

- Immediate publication on acceptance

- Inclusion in PubMed, CAS, Scopus and Google Scholar

- Research which is freely available for redistribution

Submit your manuscript at www.biomedcentral.com/submit 